\title{
Better Renal Resistive Index Profile in Subjects with Beta Thalassemia Minor
}

\author{
Fahrettin Basut $^{\mathrm{a}}$ Şakir Özgür Keşkek ${ }^{\mathrm{a}}$ Bozkurt Gülek ${ }^{\mathrm{b}}$ \\ ${ }^{a}$ Department of Internal Medicine, Numune Training and Research Hospital, Adana, Turkey; ${ }^{\mathrm{b}}$ Department of \\ Radiology, Numune Training and Research Hospital, Adana, Turkey
}

\section{Significance of the Study}

- A high level of renal resistive index has been found to be associated with arteriosclerosis. Subjects with beta thalassemia minor had lower levels of renal resistive index than healthy subjects in this study. Low renal resistive index profiles may afford some protection against cardiovascular diseases in these subjects.

\section{Keywords}

Beta thalassemia minor $\cdot$ Renal resistive index $\cdot$ Blood pressure

\section{Abstract \\ Objective: Beta thalassaemia minor is a common genetic dis- order without any characteristic symptoms except mild ane- mia. It is found to be associated with some cardiovascular risk factors such as insulin resistance and diabetes mellitus. The renal resistive index (RRI) is a measure of renal arterial resis- tance to blood flow. The aim of this study was to evaluate the $\mathrm{RRI}$ in subjects with beta thalassemia minor (BTM). Subjects and Methods: A total of 253 subjects were included in this cross-sectional study. The study group consisted of 148 sub- jects with BTM and the control group consisted of 105 healthy subjects. BTM was diagnosed by a complete blood count and hemoglobin electrophoresis. Blood pressure measurement and biochemical tests were performed. The RRI of all subjects was measured using renal Doppler ultrasonography. Results:}

Subjects with BTM had lower renal resistive indices compared to healthy subjects $(0.58 \pm 0.04$ vs. $0.60 \pm 0.06, p=$ 0.0016). Additionally, the RRI levels of subjects with BTM were correlated with systolic blood pressure ( $p=0.017, r=0.194)$. Conclusion: In this study, lower RRI were found in subjects with BTM. This may be associated with a decreased vascular resistance and blood viscosity in these subjects.

\footnotetext{
(c) 2018 The Author(s)

Published by S. Karger AG, Basel
}

\section{Introduction}

Thalassemia refers to a hereditary disease caused by a defect in $\alpha$ - or $\beta$-globin synthesis [1]. Beta thalassaemia minor (BTM) is the term applied to heterozygotes who have inherited a single gene leading to a reduced $\beta$-globin production. Subjects with BTM have no clinical problem generally, but they may show a mild hypochromic microcytic anemia. BTM is most prevalent in the Mediterra-

\section{KARGER}

E-Mail karger@karger.com www.karger.com/mpp 
Table 1. Biochemical measurements and characteristics of the subjects

\begin{tabular}{lccc}
\hline Characteristic & BTM $(n=148)$ & Healthy $(n=105)$ & $p$ \\
\hline Age, years & $35.4 \pm 11.3$ & $37.9 \pm 8.6$ & 0.06 \\
Female & $100(67.6)$ & $67(63.8)$ & 0.778 \\
BMI & $24.9 \pm 4.6$ & $24.1 \pm 2.4$ & 0.09 \\
RBC, $n \times 10^{12} / \mathrm{L}$ & $5.36 \pm 0.74$ & $4.79 \pm 0.55$ & $<0.001$ \\
Hemoglobin, g/dL & $11.7 \pm 1.8$ & $12.9 \pm 1.5$ & $<0.001$ \\
Hematocrit, \% & $36.8 \pm 5.1$ & $39.2 \pm 3.7$ & $<0.001$ \\
WBC, $n \times 10^{9} / \mathrm{L}$ & $7.2 \pm 1.9$ & $7.5 \pm 1.8$ & 0.301 \\
PLT, $n \times 10^{3} / \mu \mathrm{L}$ & $286.9 \pm 104.2$ & $295.0 \pm 81.1$ & 0.251 \\
MCV, fL & $64.9 \pm 6.4$ & $84.7 \pm 6.0$ & $<0.001$ \\
HbA,$\%$ & $4.7 \pm 0.7$ & $2.0 \pm 0.3$ & $<0.001$ \\
HBF, $\%$ & $3.62 \pm 0.73$ & $0.26 \pm 0.42$ & $<0.001$ \\
Vitamin $\mathrm{B}_{12}, \mathrm{pg} / \mathrm{mL}$ & $311.0 \pm 134.7$ & $319.6 \pm 116.3$ & 0.056 \\
Folate, $\mathrm{ng} / \mathrm{mL}$ & $8.5 \pm 3.6$ & $8.6 \pm 3.0$ & 0.437 \\
Ferritin, $\mathrm{ng} / \mathrm{mL}$ & $50.1 \pm 32.2$ & $48.4 \pm 43.1$ & 0.178 \\
\hline
\end{tabular}

Values are presented as means \pm SD or numbers (\%). BTM, beta thalassemia minor; BMI, body mass index; $\mathrm{RBC}$, red blood cells; WBC, white blood cells; PLT, platelets; $\mathrm{MCV}$, mean corpuscular volume; $\mathrm{HbA}_{2}$, hemoglobin $\mathrm{A}_{2}$; HBF, hemoglobin $\mathrm{F}$.

nean region and it is common in countries such as Turkey, Greece, Italy, and Iran $[1,2]$.

The renal resistive index (RRI), calculated on the basis of systolic and diastolic flow velocity by kidney Doppler ultrasonography, is a measure of the renal arterial resistance to blood flow [3]. The RRI has been found to be associated with arteriosclerosis and tubulointerstitial disorders. Moreover, a significant correlation persists between high levels of RRI and cardiovascular diseases $[4,5]$.

A positive correlation between RRI and insulin resistance has been shown previously $[5,6]$. Insulin resistance has been also shown in subjects with BTM [7]. Therefore, subjects with BTM may have an increased risk of atherosclerosis and cardiovascular diseases due to the insulin resistance. In this study, we aimed to investigate the level of RRI in subjects with BTM.

\section{Patients and Methods}

This case-control study was conducted in the Internal Medicine Clinic of a tertiary hospital in Turkey from January 20 to July 20, 2015. The Institutional Review Board approved this study, and informed consent was obtained from all of the subjects. The study protocol was in accordance with the Helsinki Declaration of 1975, as revised in 2008.

Two hundred fifty-three subjects participated in this study. Among them, 148 subjects had BTM and 105 were healthy subjects. Subjects with a history of a diagnosis of chronic disease, metabolic disease, or malignancy were excluded. Breastfeeding and pregnant women were also excluded.
Venous blood was collected after an 8-h fasting period. The complete blood counts, peripheral blood samples, and hemoglobin electrophoreses of all of the subjects were analyzed. Complete blood counts were measured by fluorescence flow cytometry (Sysmex XE 2100i; Japan). Serum fasting glucose and lipids were analyzed using a Beckman Coulter Synchron LX 20 (USA) using commercially available kits. Insulin levels were measured using an Abbott Architect I 2000 SR analyzer system (USA). Insulin resistance was measured using homeostasis model assessment (HOMA-IR). Ferritin, vitamin $B_{12}$, and folate levels were measured with a Roche C-601 analyzer system (Japan) using an electrochemiluminescence immunoassay. Hemoglobin electrophoresis was performed using Primus Ultra2 high-pressure liquid chromatography (USA).

The blood pressure of the patients was measured with periodically calibrated sphygmomanometers (Erka, Germany) by trained nurses. Two separate measurements were performed for each subject.

A diagnosis of BTM was established on the basis of hemoglobin $\mathrm{A}_{2}\left(\mathrm{HbA}_{2}\right) \geq 3.5 \%$, mean corpuscular volume $<80 \mathrm{fL}$, and hemoglobin $\mathrm{F}$ between 2 and $10 \%$.

The RRI of both kidneys was measured while the subject was in the supine position. All patients were examined in the morning after overnight fasting. GE Logic 9 high-resolution color Doppler ultrasonography (GE Medical Systems, Milwaukee, WI, USA) with a $5-\mathrm{MHz}$ transducer was used for the measurement of RRI by an experienced radiologist. Doppler signals were obtained from the interlober arteries. The RRI was calculated as: (peak systolic velocity - peak end diastolic velocity)/peak systolic velocity. The normal range of RRI is $0.6-0.7$.

Statistical analyses were performed using MedCalc software (version 16.8; MedCalc, Belgium). The variables were investigated using visual (histograms and probability plots) and analytical methods (Kolmogorov-Smirnov test) to determine whether they were normally distributed. Categorical measurements were reported as numbers and percentages. Quantitative measurements 
were reported as means $\pm \mathrm{SD}$. The $\chi^{2}$ test was used to compare categorical measures. The $t$ test or the Mann-Whitney $\mathrm{U}$ test was used for comparison of quantitative measurements between the 2 groups. The correlation coefficient was used for analysis of the degree of association between 2 variables (Pearson's correlation coefficient with $p$ values and 95\% CI for $r$ ). Log transformation was used for variables that were not normally distributed. OR were used for analyses of the degree of association between low levels of RRI and BTM. The probability of making a type I error ( $\alpha$, significance) was 0.05 in all of the tests.

\section{Results}

There were no statistically significant differences in the age of the the BTM (study) and healthy (control) groups ( $p=0.06$ and $p=0.778$, respectively). Demographic and laboratory data of the groups are shown in Table 1.

There were statistically significant differences between the groups in terms of $\mathrm{HbA}_{2}$ and hemoglobin F. Subjects with BTM had higher levels of both $\mathrm{HbA}_{2}$ and hemoglobin $\mathrm{F}(p<0.001$, respectively). The mean corpuscular volume was lower in subjects with BTM $(p<0.001)$. Hemoglobin and hematocrit levels were lower $(p<0.001)$ and RBC levels were higher $(p<0.001)$ in subjects with BTM. Platelet, white blood cells, ferritin, vitamin $B_{12}$, and folate levels were not significantly different in the 2 groups $(p>$ 0.05 , respectively; Table 1 ).

Fasting glucose, insulin, and HOMA-IR were higher in subjects with BTM ( $p=0.009, p=0.003$, and $p=0.001$, respectively; Table 2). Serum triglyceride, LDL, HDL, and creatinine levels were comparable in both groups (Table $2)$. Systolic blood pressure was lower in subjects with BTM $(p=0.017)$. Diastolic blood pressures were not significantly different ( $p=0.322$; Table 3$)$. The mean RRI was $0.58 \pm 0.04$ in subjects with BTM and $0.60 \pm 0.06$ in healthy subjects. Subjects with BTM had significantly lower RRI levels than those in the healthy group $(p=$ 0.0016; Table 3).

RRI levels of subjects with BTM were correlated with systolic blood pressure ( $p=0.017, r=0.194$; Fig. 1$)$. There was a strong relationship between low RRI and BTM (OR 4.96; 95\% Cl 2.41-10.2, $p<0.001$ ).

\section{Discussion}

In the current study, we investigated the level of RRI in subjects with BTM. We compared the BTM subjects with a frequency matched healthy group. As a common
Table 2. Metabolic parameters of the subjects

\begin{tabular}{lccl}
\hline & $\begin{array}{l}\text { BTM } \\
(n=148)\end{array}$ & $\begin{array}{l}\text { Healthy } \\
(n=105)\end{array}$ & $p$ \\
\hline Fasting glucose, mg/dL & $94.7 \pm 10.1$ & $91.4 \pm 9.5$ & 0.009 \\
Fasting insulin, $\mu \mathrm{U} / \mathrm{mL}$ & $13.1 \pm 7.7$ & $11.1 \pm 7.2$ & 0.003 \\
HOMA-IR & $3.02 \pm 1.84$ & $2.51 \pm 1.77$ & 0.001 \\
HDL, mg/dL & $47.6 \pm 11.6$ & $49.3 \pm 9.4$ & 0.08 \\
LDL, mg/dL & $103.1 \pm 39.3$ & $106.0 \pm 34.9$ & 0.276 \\
Triglyceride, mg/dL & $132.8 \pm 43.8$ & $126.7 \pm 50.8$ & 0.127 \\
Creatinine, mg/dL & $0.67 \pm 0.16$ & $0.65 \pm 0.12$ & 0.134 \\
\hline
\end{tabular}

Values are presented as means \pm SD. HOMA-IR, homeostasis model assessment of insulin resistance; BTM, beta thalassemia minor; HDL, high-density lipoprotein; LDL, low-density lipoprotein.

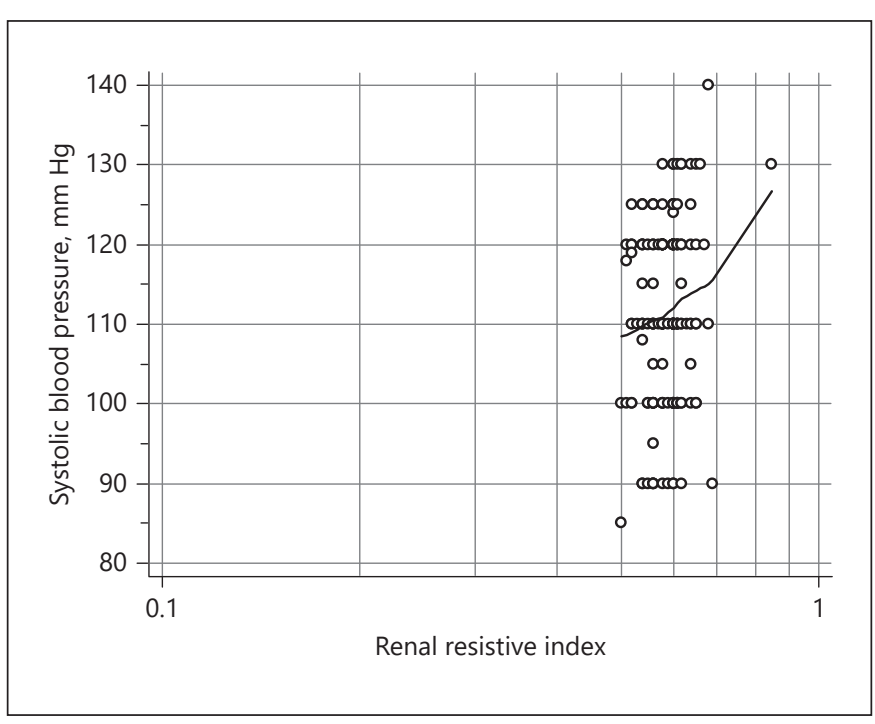

Fig. 1. Correlation between systolic blood pressure and renal resistive index of subjects with beta thalassemia minor.

and mostly symptomless disease [2], BTM is usually diagnosed when a complete blood count has been obtained for other reasons. Besides, BTM is found to be associated with some cardiovascular risk factors such as insulin resistance and diabetes mellitus $[7,8]$. On the other hand, RRI is a good indicator of intrarenal hemodynamics and it has been also found to be associated with diabetes mellitus and insulin resistance $[5,6,9]$.

We found low levels of RRI in subjects with BTM in this study. High RRI have been demonstrated previously in patients with hypertension. In a study by Keşkek et al. [5], 116 patients with hypertension were compared with 105 healthy subjects; the authors found higher RRI in pa- 
Table 3. Blood pressure and renal resistive index of the subjects

\begin{tabular}{lccc}
\hline & $\begin{array}{l}\text { BTM } \\
(n=148)\end{array}$ & $\begin{array}{l}\text { Healthy } \\
(n=105)\end{array}$ & $p$ \\
\hline Systolic blood pressure, mm Hg & $111.4 \pm 11.4$ & $\begin{array}{c}115.0 \pm 12.5 \\
71.6 \pm 11.4\end{array}$ & $\begin{array}{l}0.017 \\
0.322 \\
\text { Diastolic blood pressure, mm Hg }\end{array}$ \\
$\begin{array}{l}70.3 \pm 9.4 \\
\text { Renal resistive index }\end{array}$ & $0.58 \pm 0.04$ & $0.60 \pm 0.06$ & 0.0016 \\
\hline
\end{tabular}

Values are presented as means \pm SD. BTM, beta thalassemia minor. tients with hypertension [5]. Kawai et al. [10] reported high values of RRI in a group of 143 hypertensive patients; similarly, Trovato et al. [11] reported high RRI in 119 hypertensive individuals compared to 150 subjects with a normal blood pressure. Vascular resistance, vascular compliance, atherosclerosis, and vessel damage are important predictors of RRI and they are associated with hypertension.

We also found lower levels of systolic blood pressure in subjects with BTM. A lower risk of areterial hypertension in subjects with BTM can be caused by hemodynamic variations such as arterial dilatation and lower blood viscosity. Decreased vascular resistance due to the arterial dilatation may develop in subjects with anemia. Besides, decreased blood viscosity due to the decreased hematocrit level can improve circulation $[12,13]$. In accordance with this link, hypertensive patients with BTM presented with a better 24-h blood pressure profile compared to patients without BTM [14]. Baserga et al. [15] reported a lower incidence of hypertension in subjects with BTM. Additionally, a high hemotocrit percentage and policythemia have been reported to be associated with a high blood pressure in other studies $[12,16]$.

We found high levels of fasting glucose, insulin, and HOMA-IR in subjects with BTM compared to healthy subjects. Chronic hemolysis of microcytic erythrocytes leads to increased oxidative stress and liver inflamma- tion in subjects with BTM. This pathophysiologic mechanism may be responsible for insulin resistance and diabetes in these subjects [8]. A study on 82 BTM subjects and 82 healthy subjects showed that the development of diabetes and insulin resistance in subjects with BTM was 2 times greater than in the general population [8]. Additionally, high fasting insulin levels and insulin resistance have been reported in BTM subjects with a normal glucose tolerance [17] and high levels of fasting glucose and insulin levels have been shown in subjects with BTM [17].

Our study has some limitations. First, it would have been beneficial if the genetic analyses had been done for the diagnosis of BTM. Second, the ambulatory blood pressure of the patients was not measured. Further, the cross-sectional design of this study may be another limitation.

In conclusion, we found lower levels of RRI in subjects with BTM in this study. Subjects with BTM have low RRI due to the low systolic blood pressure. BTM may afford some protection against cardiovascular diseases due to the low RRI and systolic blood pressure.

\section{Disclosure Statement}

None.

\section{References}

1 Giardina PJ, Forget BG: Thalassemia syndromes; in Hoffman R, Benz EJ Jr, Shattil SJ, et al (eds): Hematology: Basic Principles and Practice. New York, Churchill Livingstone, 2009, vol 5, pp 555-557.

2 Benz EJ: Disorders of hemoglobin; in Kasper DL, Fauci AS, Hauser SL, Longo DL, Jameson JL, Loscalzo J (eds): Harrison's Principles of Internal Medicine. New York, McGraw-Hill, 2015, vol 19, pp 637-639.
3 Ikee R, Kobayashi S, Hemmi N, et al: Correlation between the resistive index by Doppler ultrasound and kidney function and histology. Am J Kidney Dis 2005;46:603-609.

4 Doi Y, Iwashima Y, Yoshihara F, et al: Renal resistive index and cardiovascular and renal outcomes in essential hypertension. Hypertension 2012;60:770-777.
5 Keşkek ŞÖ, Çinar Y, Kirim S, et al: High renal resistive index in hypertensive patients is also associated with serum homocysteine level. Clin Exp Nephrol 2015;19:639-645.

6 Afsar B, Elsurer R, Sezer S, et al: Insulin resistance is associated with increased renal resistive index independent of other factors in newly diagnosed type 2 diabetes mellitus and hypertensive patients. Metabolism 2010;59: 279-284. 
7 Kırım S, Keşkek şÖ, Turhan A, et al: Is $\beta$-thalassaemia minor associated with metabolic sisorder? Med Princ Pract 2014;23:421425.

8 Bahar A, Kashi Z, Sohrab M, Kosaryan M, Janbabai G: Relationship between beta-globin gene carrier state and insulin resistance. J Diabetes Metab Disord 2012;11:22.

9 Gaipov A, Solak Y, Zhampeissov N, et al: Renal functional reserve and renal hemodynamics in hypertensive patients. Ren Fail 2016;38: 1391-1397.

10 Kawai T, Ohishi M, Kamide K, et al: The impact of visit-to-visit variability in blood pressure on renal function. Hypertens Res 2012; $35: 239-243$
11 Trovato GM, Pace P, Martines GF, et al: Stress, abdominal obesity and intrarenal resistive index in essential hypertension. Clin Ter 2012;163:299-305.

12 Sloop G, Holsworth RE Jr, Weidman JJ, St Cyr JA: The role of chronic hyperviscosity in vascular disease. Ther Adv Cardiovasc Dis 2015; 9:19-25.

13 Karimi M, Marvasti VE, Motazedian S, et al: Is beta-thalassemia trait a protective factor against hypertension in young adults? Ann Hematol 2006;85:29-31.
14 Vyssoulis G, Karpanou E, Kyvelou SM, et al: Ambulatory blood pressure profile in hypertensive patients with $\beta$-thalassemia minor Hypertens Res 2011;34:253-256.

15 Baserga A, Barrai I, Bonomo L, et al: Clinical aspects of beta-thalassaemia minor. Panminerva Med 1982;24:275-277.

16 Namazi MR: Minor thalassemia as a protective factor against cerebrovascular accidents. Med Hypotheses 2002;59:361-362.

17 Tong PC, Ng MC, Ho CS, et al: C-reactive protein and insulin resistance in subjects with thalassemia minor and a family history of diabetes. Diabetes Care 2002;25:1480-1481. 